METHODS/STUDY POPULATION: First stage of the training sessions (TS) dealt with the theory of CTR. After TS and responding to their research interests, as answered in a questionnaire, the participants formed a CTMT, under the mentorship of a well-established CT researcher. This, as a prelude to their hands-on experiences in Intensive Development and Experiences in Advancement of Research and Increased Opportunities (IDEARIO), for which a research proposal is needed. RESULTS/ANTICIPATED RESULTS: Five (5) CTMTs were formed in different research areas - cardio, neuro, liver, renal, Zika-, as submitted in their research concept papers.Eight (8) CT researchers are currently mentoring 2 US, 7 GS and $6 \mathrm{~F}$ of HSPs through the CTMTs. They have submitted a research proposal, as a bridge between the theory in the TS and the practice in IDEARIO. Five (5) proposals were received and 2 of them approved, while the other 3 are in the evaluation process. We will present the composition, research topics, development of research and the feedback of participants in IDEARIO and CTMTs. DISCUSSION/SIGNIFICANCE OF IMPACT: The CTMTs and their respective proposals are effective strategies for the mentoring of US, GS and F in CTR.

\section{Clinical research training methods that improve self-efficacy in clinical research domains}

Mathew Sebastian ${ }^{1}$, Matthew Robinson ${ }^{1}$, Leanne Dumeny ${ }^{1}$, Kyle Dyson, Wayne T. McCormack ${ }^{1}$ and William Stratford May

${ }^{1}$ University of Florida Clinical and Translational Science Institute

OBJECTIVES/SPECIFIC AIMS: The study aims to determine the current clinical research training interventions of $\mathrm{MD}-\mathrm{PhD}$ programs and how effective they are in promoting clinical research self-efficacy. METHODS/STUDY POPULATION: A national survey of $\mathrm{MD}-\mathrm{PhD}$ trainees was conducted in 2018 to identify clinical research training methods and self-efficacy for clinical research skills. MD-PhD program directors and coordinators from 108 institutions were asked to distribute the survey to their students. Responses were received from 61 institutions (56.5\%). Responses were obtained from $647 \mathrm{MD}-\mathrm{PhD}$ students in all years of training, representing $17.9 \%$ of the 3613 possible participants at the 61 medical schools represented. No compensation was provided for this study. RESULTS/ANTICIPATED RESULTS: The primary methods of clinical research training reported by students included didactics, mentored clinical research, didactics plus mentored clinical research, didactics plus clinical research practicum, and didactics plus mentored clinical research plus clinical research practicum. A quarter of all participants reported having no clinical research training. Clinical research self-efficacy was then correlated with the amount of clinical research training. Students exposed to no clinical research had the lowest self-efficacy in clinical research skills and students experiencing didactics plus mentored clinical research plus clinical research practicum had the highest perceived self-efficacy in clinical research domains. DISCUSSION/SIGNIFICANCE OF IMPACT: This is one of the first studies assessing clinical research training methods for MD-PhD students and assessing their efficacy. We found that of all students questioned, 25\% mentioned had not received any type of clinical research training. The remaining students identified 5 research training methods that institutions currently use. This work highlights the importance of clinical research experience students need to improve their self-efficacy, a major influence on research career outcomes.

3366

\section{Communication in Science: a summer workshop program at Mount Sinai}

Janice Lynn Gabrilove, MD, FACP ${ }^{1}$ and Layla Fattah ${ }^{1}$

${ }^{1}$ Mount Sinai School of Medicine

OBJECTIVES/SPECIFIC AIMS: In an effort the increase awareness and enhance knowledge and skills in relation to communication in science at Mount Sinai, the Communication in Science summer workshop series aimed to provide an accessible, workforce-wide lecture series to promote key concepts and skills related to communicating science. Delivered by faculty and invited speakers, a series of seven workshops delivered over a 4 week period covered topics such as communication in teams, storytelling and TED talk principles, and community engagement. The aim of each session was to offer "top tips" that participants could apply to their practice. Evaluation of the workshop series aimed to determine participant satisfaction and self-perceived changes in knowledge and skills in relation to science communication. METHODS/STUDY POPULATION: A total of 375 participants registered to attend the workshop series from a range of backgrounds including post-docs, faculty, residents, staff and students at Mount Sinai. Attendance at the workshops ranged from a high of 119 and a low of 33 participants, with as many as $41 \%$ of attendees joining the session via live-streaming. Participants were emailed an online survey at the end of the workshop series, asking for satisfaction feedback on each individual workshop and an overall impression of the workshop series. Participants were asked to rate the satisfaction criteria related to content, gained knowledge and skills, presentation style and whether they found the session of value for each workshop, using a Likert scale from $1-5$ ( 1 = strongly disagree, $5=$ strongly agree). Participants were also asked to provide an overall rating for the summer workshop series as a whole. RESULTS/ ANTICIPATED RESULTS: A total of 35 participants responded to the survey. Mean responses to the survey questions were:. The content of this session is important to my work $=4.09$ (range $3.77-4.45$ ). This session increased my knowledge or skills 4.03 (range 3.56- 4.62). The presenters delivered this content clearly $=4.16$ (range $3.78-$ 4.67). Overall I found this session valuable $=4.13(3.78-4.61)$ Participants were also asked to provide an overall rating for the summer workshop series as a whole on a scale of 1 to 10 ( $1=$ poor, $10=$ excellent). The mean response was 8.36 , indicating a high level of satisfaction with the program. Qualitative feedback indicated that the sessions were successful in increasing awareness of this topic. One participant reported that "these sessions inspired me to think differently, and in a way that can potentially allow me to communicate with the non-science community". DISCUSSION/ SIGNIFICANCE OF IMPACT: The high number of registrants for this summer workshop series indicates a perceived need for education and training on Communication in Science at Mount Sinai. Sessions that focused on TED talk principles and storytelling in science were particularly well attended and well-reviewed, suggesting a particular interested in these topics. There was, however, a discrepancy between registration and attendance numbers, which going 